\title{
The Impact of the Serotonin Transporter Gene Promoter DNA Methylation on Anorexia Nervosa囚A Preliminary Longitudinal Study
}

Jue Chen ( $\square$ chenjue2088@163.com )

Shanghai Jiao Tong University School of Medicine

\section{Zeping Xiao}

Shanghai Mental Health Center, Shanghai Jiao Tong University School of Medicine

Qianqian He

Shanghai Mental Health Center, Shanghai Jiao Tong University School of Medicine

Qing Kang

Shanghai Mental Health Center, Shanghai Jiao Tong University School of Medicine

\section{Shunying Yu}

Shanghai Mental Health Center, Shanghai Jiao Tong University School of Medicine

\section{Sufang Peng}

Shanghai Mental Health Center, Shanghai Jiao Tong University School of Medicine

Han Chen

Shanghai Mental Health Center, Shanghai Jiao Tong University School of Medicine

Zhiguang Lin

Shanghai Mental Health Center, Shanghai Jiao Tong University School of Medicine

\section{Research Article}

Keywords: anorexia nervosa, SLC6A4 promoter gene DNA methylation, 5-HTTLPR, epigenetics, therapeutic response

Posted Date: December 16th, 2021

DOI: https://doi.org/10.21203/rs.3.rs-1118582/v1

License: (c) (i) This work is licensed under a Creative Commons Attribution 4.0 International License. Read Full License 


\section{Abstract}

\section{Backgroud}

The serotonin system has been reported to be involved in the pathogenesis of anorexia nervosa (AN). The promoter region of serotonin transporter gene (SLC6A4), also called 5-HTTLPR, responsible for serotonin reuptake, has received much attention, especially 5-HTTLPR methylation, which was associated with abnormal eating behaviors. The study was aimed to explore the association between 5-HTTLPR methylation and $\mathrm{AN}$, as well as its predictive effect on therapeutic response.

\section{Methods}

91 AN patients and 87 healthy controls (HCs) were recruited. Only 30 patients completed the 12-week follow-up. 5-HTTLPR methylation levels and clinical symptoms were assessed at baseline for all participants, at the fourth and the twelfth week for follow-up patients. 5-HTTLPR methylation was measured by MassArray methods and clinical symptoms were mainly evaluated by the EDE-Q6.0 scale.

Results

AN patients had higher methylation at $\mathrm{CpG} 11, \mathrm{CpG} 24.25, \mathrm{CpG} 31.32$ and CpG_mean than healthy controls $(P=0.039,0.042,0.018,0.001)$. Furthermore, it was the binge/purging subtype that revealed significant hypermethylation at CpG4 and CpG_mean $(P=0.027,0.031)$. However, it failed to discover significant differences between AN-R and AN-BP groups at all units. Besides, CpG 11 methylation level was positively correlated with EDEQ-6.0 total score in patients $(r=0.226, P=0.047)$. The methylation level of CpG11, CpG26.27.28, CpG31.32, CpG33.34.35.36 and CpG_mean decreased after treatment, but not significantly. 5-HTTLPR methylation was not significantly different between the two groups after treatment.

\section{Conclusions}

Our study provided preliminary evidence that 5-HTTLPR methylation played vital roles in AN pathogenesis. The characteristic of 5-HTTLPR among untreated AN patients was hypermethylation. However, whether it is the biomarker to distinguish the subtypes and therapeutic response of AN needs further investigation.

\section{Plain English Summary}

Anorexia nervosa (AN) is a severe, often chronic eating disorder (ED) with a high mortality rate especially in adolescent females. It includes two subtypes of restricting type (AN-R) and binge/purging type (AN-BP) that is difficult to treat. Patients often face great physical and psychological suffering due to various physical complications resulting from malnutrition. Anorexia nervosa is an important cause of physical and psychosocial morbidity. In recent years, epigenetics research has received much attention, especially DNA methylation. Many studies have reported the relationship between the serotonin system and 
abnormal eating behaviors in AN. The promoter region of serotonin transporter gene (SLC6A4), also called 5-HTTLPR, has received much attention. In our study, it found untreated AN patients had higher methylation at 5-HTTLPR than healthy controls, indicating its vital roles in AN pathogenesis. Further research needs to investigate the effect of 5-HTTLPR methylation on therapeutic response and explore its treatment role in AN patienrs.

\section{Introduction}

Anorexia nervosa (AN) is one of the most severe and complex eating disorders (EDs) characterized by strict restrictions of energy intake, a strong desire to stay extremely thin, a pathological fear of weight gain combined with a distorted body perception, which mainly affects young women [1]. AN patients are divided into restricting type (AN-R) and binge/purging type (AN-BP) based on whether they had binge eating and vomiting behaviors in the diagnostic and Statistical Manual of Mental Disorders (DSM-V) [2]. These symptoms make patients unable to comprehend the seriousness of the illness, as a result, they stay a pathological state persistently, which leads to serious distress and burden for their family and society. Especially, the prevalence of the disease is rising continuously in China and has become comparable to that worldwide [3]. Growing interest has focused on the pathogenesis and treatment of AN.

AN is believed to be the product of Gene $x$ environment $(\mathrm{GxE})$ interaction, which plays a vital role in the psychology of EDs [4]. The research is still relatively novel, among which DNA methylation is the most widely studied. DNA methylation is one of the most crucial epigenetic modifications, mediated by environmental effects on gene expression without changing DNA sequences [5]. It is a process in which a methyl group is added to the nucleotide base pair cytosine next to a guanine, that happens at the specific dinucleotide units called CpG islands (CpGs), leading to reduced gene expression [6].

The serotonin system has been reported to be involved in the pathogenesis of anorexia nervosa[7, 8]. The serotonin transporter gene (SLC6A4), also known as serotonin transporter (5-HTT), located on human chromosome 17q11.1-q12[9], coding for the 5-HTT serotonin transporter protein, was supposed to be implicated in EDs [10]. It also acts a key role in regulating eating behaviors by controlling hunger and promoting satiety and being highly influenced by nutritional intake [11]. Several animal studies remained the role of $5-\mathrm{HT}$ in the inhibition of feeding behaviors, which could promote satiety and produce an anorexic effect $[12,13]$.

Serotonin Transporter Linked-Polymorphic Region (5-HTTLPR), located at the promoter region of the SLC6A4 gene, is one of the most investigated polymorphisms[14], which was discovered to impact the transcriptional activity of serotonin and regulate its expression[15]. There is some evidence that the 5HTTLPR genotype affects vulnerability to a broad range of mental disorders including depression [16], panic disorder (PD) [17], post-traumatic stress disorder (PTSD) [18], and so forth. However, scarce studies have been performed in ED patients and longitudinal studies in AN have not yet been investigated. SLC6A4 DNA methylation could result in reducing 5-HTT gene expression, which was reported to be 
associated with abnormal eating behaviors such as food restriction and food avoidance in the previous study [19]. Thus, we make a high hypothesis that SLC6A4 DNA methylation acts a critical part in restrictive eating and AN pathogenesis.

This study provides a novel perspective to help us understand the epigenetic mechanism of 5-HTTLPR methylation for AN. This is the first investigation to explore whether there is association between 5HTTLPR methylation level and AN, as well as clinical symptoms before and after treatment in AN patients. We expect that DNA methylation will be associated with $\mathrm{AN}$, together with clinical symptoms and therapeutic response of AN.

\section{Methods}

\section{Study design}

AN patients in this study came from two different projects. The methylation of 5-HTTLPR and clinical symptoms were assessed at the baseline for all patients, however, only thirty patients from one project completed the subsequent follow-up. The methylation level of 5-HTTLPR were measured at the twelfth week and clinical symptoms were evaluated at the fourth and twelfth week for follow-up patients. Meanwhile, they received routine outpatient treatment, mainly including physical and mental examinations, psychological counseling, nutritional evaluation, and drug treatment based on their situations. There is no required treatment in our study. The study protocol and process were approved by the ethics committee of the Shanghai Mental Health Center.

\section{Participants}

A total of ninety-one AN patients aging 13-60 years were recruited from the department of psychological outpatient and inpatient in Shanghai Mental Health Eating Disorder Treatment Center. All patients were assessed and diagnosed by two senior psychiatrists according to DSM-5 (American Psychiatry Association). Each of them met the following inclusion and exclusion criteria: (a) female, Chinese Han, Junior high school or above; (b) BMI from $13.0 \mathrm{~kg} / \mathrm{m}^{2}$ to $18 \mathrm{~kg} / \mathrm{m}^{2}$, no serious or chronic somatic disease; (c) no history of another psychiatric disorder; (d) have not taken psychiatric and hormone drugs since the illness onset or at least for twelve weeks; (e) not currently pregnant or breastfeeding; $(f)$ capable of performing all tests and signed informed consent. These patients included AN-R subtypes $(n=44)$ and AN-BP subtypes $(n=47)$. Eighty-seven healthy controls $(H C)$ were recruited among colleges and health care workers through advertisements. The inclusion and exclusion criteria were in accordance with those of AN patients, except that they were not diagnosed with anorexia nervosa on the basis of DSM- V.

\section{Clinical assessment}

At the baseline, demographic information such as age, gender, education level, as well as family history, psychological characteristics and clinical features of all participants were collected. The follow-up 
patients needed to be assessed the psychological characteristics and clinical features at the fourth and twelfth week, which were measured using the following instruments:

\section{Eating Attitudes Test-26 (EAT-26)}

The scale is a 26 -item, self-screening questionnaire used to assess the symptoms and attitudes of ED patients. It mainly consists of three dimensions: diet, gluttony, food preoccupation concept and oral control. Its standards have been evaluated in Hong Kong [20]. Our previous results preliminarily confirmed that the reliability and validity of the scale in mainland China are good with internal consistency of 0.910 , test-retest reliability of 0.817 , convergent validity of 0.744 , specificity of 0.85 and approximate sensitivity of 0.66 [21].

\section{Questionnaire Version of the Eating Disorders Examination (EDE-Q 6.0)}

The scale is a 28-item, self-report questionnaire widely used to assess the severity of ED, that consists of four subscales: restricted eating, attention to eating, attention to body shape, and attention to weight. The Chinese version of the scale was provided by Professor Li of Hong Kong University, whose reliability and validity were good with internal consistency of 0.95 , test-retest reliability of 0.79 , and convergent validity of $0.72[22]$. In addition, our previous study preliminarily confirmed that the reliability and validity of the scale is good in mainland China [23].

\section{Other questionnaires}

In addition to the above scales, some other rating scales were also included. Hamilton Depression Rating Scale (HAMD), Hamilton Anxiety Scale, (HAMA) and Yale Brown Obsessive Compulsive Scale (YB-OCS) were adopted to evaluate the depression, anxiety and compulsive symptoms respectively in AN patients.

\section{SLC6A4 promoter methylation}

Venous blood samples were obtained from all participants and collected by EDTA tubes. DNA was extracted using a DNA extraction kit (Tiangen, China) and then purified and converted by bisulfite using a EZ DNA Methylation Kit ${ }^{\mathrm{TM}}$ protocol (Zymo Research, USA). The promoter-associated CpG island was targeted in our assay, primers of which were used to amply bisulfite treated DNA. The primers for SLC6A4 were designed by the online software EpiDesigner webunit (www.epidesigner.com, Agena Bioscience, USA), that were as follows: forward 5'-aggaagagagTTGTTAGGTTTTAGGAAGAAAGAGAGA-3' and reverse 5'-cagtaatacgactcactatagggagaagg ctCCCTCACATAATCTAATCTCTAAATAACC-3', a T7-promoter tag was attached to the reverse primer for the subsequent PCR amplification. The reaction conditions for the PCR were as follows: $94 \circ \mathrm{C}$ for $4 \mathrm{~min}$, followed by 45 cycles at $94 \circ \mathrm{C}$ for $20 \mathrm{sec}, 56 \circ \mathrm{C}$ for $30 \mathrm{sec}, 72 \circ \mathrm{C}$ for $1 \mathrm{~min}$, then $72 \circ \mathrm{C}$ for $3 \mathrm{~min}$. The PCR products were treated with shrimp alkaline phosphatase (SAP), followed by uracil- specific cleavage reaction using Mass CLEAVE ${ }^{\mathrm{TM}}$ Reagent Kit (Agena, USA). The Agena Mass ARRAY platform was used to analyze the methylation levels of each CpG island in the SLC6A4 gene promoter. Methylation data were generated by the EpiTYPER software v1.2(Agena, USA). The specific principle was shown in the figure 1. 


\section{Statistical analysis}

All statistical analysis were conducted by SPSS statistical analysis software (version 22.0; IBM SPSS, Chicago, IL, USA) and GraphPad Prism 8.0 (https://www.graphpad.com/guides/prism/8/userguide/index.html, accessed on 25 April 2021). The data were expressed as numbers and frequencies for categorical variables and analyzed by $x 2$-test. The continuous variables that were normally distributed are presented as mean \pm standard and differences between them were determined by $T$ test and one-way analysis of variance (ANOVA). While the variables that were not normally distributed are presented as the median (maximum and minimum), and differences between them were determined by Mann-whitney $U$ test and Kruskal-Wallis ANOVA test. Apart from the methylation level of CpG4 unit in 5-HTTLPR, others were all normally distributed. Pearson correlation analysis (normal distribution data) and Spearman correlation analysis (non-normal distribution data) were adopted to analyse the association between DNA methylation level in 5-HTTLPR and clinical features. Repeated-measures ANOVAs were used to compare the changes of DNA methylation levels in 5-HTTLPR before and after treatment. All tests were two-tailed and $p$-values $<0.05$ were considered to be statistically significant.

\section{Results}

\section{Demographic and clinical characteristics}

Ninety-one AN patients without treatment and eighty-seven HCs completed the study. All participants were female with mean age of $18.96 \pm 4.14$ years old and mean education level of $12.18 \pm 2.94$ years. The mean age of AN onset was 16.5 \pm 2.64 years old and the mean course of AN was 15 years. As shown in Table 1, there were no significant differences in age $(P=0.068)$ and education level $(P=0.062)$ between the two groups. The current BMI index, ideal body weight and ideal BMI index of AN patients were significantly lower than those of $\mathrm{HC}$ group $(P<0.05)$. But no significant differences were found in the maximum body weight $(P=0.820)$ and maximum BMI $(P=0.531)$ between the two groups. Clinical characteristics have significantly higher scores in EAT-26 and EDE-Q6.0 scales $(P<0.05)$, the abnormal eating symptoms of AN patients were more serious than that of $\mathrm{HC}$ groups.

\section{Differences of DNA methylation levels in 5-HTTLPR in AN patients and healthy controls}

All CpG units of the 5-HTTLPR methylation level were analyzed by MassArray Epityper mass spectrometer and quantified as percentage. The amplified DNA fragment of each sample contained 7 CpG units, among which more than $50 \%$ of GpG3 units failed to be detected successfully, so they were not analyzed, six units were finally included in the statistics. Through intergroup comparison, the methylation level of the $\mathrm{CpG11}$, CpG24.25, CpG31.32 and CpG_mean in AN group was found to be significantly higher than those in $\mathrm{HC}$ group $(P=0.039,0.042,0.018,0.001$ respectively). See Figure 2 .

\section{Differences of DNA methylation levels in 5-HTTLPR in different subtypes of AN patients and healthy controls}


AN patients were further divided into restricting $(n=44)$ and binge-eating/purging subtypes $(\mathrm{n}=47)$. Through Kruskal-wallis ANOVA test, it showed significant differences in CpG24.25 and mean methylation level among AN-R, AN-BP and $\mathrm{HC}$ groups $(P=0.008)$. Further, the methylation level of CpG24.25 and CpG_mean in AN-BP group was significantly higher than that of $\mathrm{HC}$ groups $(P=0.027$, 0.031 respectively). However, there was no significant differences between AN-R group and AN-BP group $(P>0.05)$, AN-R group and HC group ( $P>0.05)$. See Figure 3.

\section{Association analysis between DNA methylation level in 5-HTTLPR and clinical features before treatment in AN patients}

According to correlation analysis, it was found that the methylation level of CpG11 unit was positively correlated with the total score of EDE-Q6.0 scale $(r=0.216, P=0.047)$. No significant correlation was found between the methylation level of all units in SLC6A4 gene and depression, anxiety and obsessivecompulsive symptoms of AN patients. See Table 2.

\section{Changes in clinical symptoms and methylation level of 5-HTTLPR after treatment in AN patients}

Only thirty patients completed the follow-up of twelve weeks. Comparing the clinical symptoms and methylation level before and after treatment by repeated-measures ANOVA, it was found that after fourth and twelfth week treatment, BMI index significantly increased (P冈0.05), the total score of HAMD, HAMA, YB-OCS scale, both the total score and factor score of EAT-26, and EDE-Q6.0 scale significantly decreased in AN patients ( $P \otimes 0.05)$. In addition, it revealed that the methylation level of CpG11, CpG26.27.28, CpG31.32, CpG33.34.35.36 units and CpG_mean decreased after twelve weeks treatment, but no significant differences were found $(P \otimes 0.05)$. See Table 3.

\section{Association analysis between the change of DNA methylation level in 5-HTTLPR and the change of clinical features before and after treatment in AN patients.}

The DNA methylation level of 5-HTTLPR decreased after 12 weeks treatment compared with basement in AN patients. The changes of each point were described as res_CpG. Similarly, the changes of EDEQ-6.0 total score were described as res_EDEQ-6.0. Correlation Analysis between the change of 5-HTTLPR methylation level and the change of clinical symptoms after treatment showed that the res_CpG1 was significantly positively correlated with res_EDEQ-6.0 ( $r=0.556, P=0.028)$. However, further considering age, onset age, education level, disease course and other factors as covariables, the influence of CpG1 unit was no longer significant $(\mathrm{F}=0.908, P=0.409)$. No correlation was found between the changes of other units and clinical symptoms $(P \otimes 0.05)$.

\section{Discussion}

Our study adopted preliminarily cross-sectional and longitudinal design to investigate the possible association between 5-HTTLPR methylation level and AN, as well as clinical symptoms before and after treatment in AN patients. We examined seven $\mathrm{CpG}$ units of 5-HTTLPR, among which six CpG 
units were detected except $\mathrm{CpG} 3$ unit. AN were significantly associated with higher methylation at $\mathrm{CpG} 11$, CpG24.25, CpG31.32, suggesting that 5-HTTLPR methylation may contribute to AN pathogenesis. Moreover, the methylation level of CpG24.25 unit and CpG_mean in AN-BP groups, not the AN-R groups were significantly higher than that in $\mathrm{HC}$ groups. Further, the study failed to discover significant difference of all units between AN-R group and AN-BP group, so the 5-HTTLPR methylation might be not a biomarker to distinguish the two subtypes of AN. Meanwhile, positive correlations were reported between CpG11 methylation level and the total score of EDE-Q6.0 scale.

From the perspective of longitudinal studies, abnormal eating and emotional state, the clinical symptoms improved in patients after the fourth and twelfth week of treatment, and the methylation level of CpG11, CpG26.27.28, CpG31.32, CpG33.34.35.36 units and CpG_mean decreased, but not significantly, after twelve weeks treatment. In addition, no significant correlations were found between the change of DNA methylation level and the change of clinical features after treatment in patients.

Studies have confirmed that 5-HTTLPR methylation level in peripheral blood lymphocyte was associated with that in brain [24], and a majority of epigenetics did research using the peripheral blood lymphocyte samples[25, 26], which provide a theoretical basis for our study. Our study showed a significant association between SLC6A4 methylation and AN, which was in concordance with the observation in patients with depression [26, 27]. Probably because SLC6A4 hypermethylation causes the decrease of mRNA expression, which leads to the decrease of 5-HT transporter protein and 5-HT reuptake, then resulting in the increase of 5-HT concentration in synaptic space and the decrease of 5-HT synthesis by negative feedback, finally it may increase the efficacy of serotonergic antidepressants[28]. While previous study did not find positive results in AN patients [29], which may be due to the differences in the samples and fragments selected. According to our results, the methylation level of CpG11, CpG24.25 and CpG26.27.28 units may be related to AN. Besides, the methylation level of 5-HTTLPR was connected with eating symptoms but not the accompanying emotional symptoms, indicating it may play a specific role in AN. Since there are no studies to explore the effect of 5-HTTLPR methylation on the clinical symptoms of AN patients, the conclusion needs to be verified from other perspectives, like the brain function[25, 30].

Long-term follow-up study on patients with different subtypes of AN showed that most AN started with AN-R, about $88 \%$ of AN-R can develop bulimic behaviors, $62 \%$ of AN-R eventually develops into AN-BP subtype [31]. To some extent, different subtypes of AN can be seen in different stages of disease development. Our study showed AN-BP patients had higher methylation than healthy controls, probably indicating alterations in trait-related serotonergic function, which might be related with 5-HTT [32]. The differences failed to be found in AN-R patients, may be due to the absence of significant changes in biogenetic indicators in the acute phase of AN-R patients.

Considering the possible effect of 5-HTTLPR methylation on long-term AN outcome, some studies have reported the predictive effect of 5-HTTLPR methylation on the therapeutic response in depression patients, the higher the 5-HTTLPR methylation level, the better the antidepressant response[28, 33], but there were also contrary results[34], as for how gene methylation affects the therapeutic effect has not 
been reported. It was the methylation level of CpG11 unit in 5-HTTLPR that was significantly associated with therapeutic responses[35]. However, it was not consistent in our study, which may due to the different mechanisms of SLC6A4 gene in the two diseases, in addition, it is hard to detect the association between the SLC6A4 gene methylation and treatment response in a short time for the chronicity and obstinacy of AN. Thus, it may not be suitable as a biomarker to evaluate the outcome of AN.

Few previous studies conducted on 5-HTTLPR in patients with EDs. One of which indicated that the S-

allele of the 5-HTTLPR genotype particularly increased the risk susceptibility for AN and was related to its instability and depressive comorbidity[32]. Thus, a study has reported that the effects of 5-HTTLPR genetic variants may contribute to the regulation in modifying its methylation [36], which has been validated in patients with depression[26, 28] and anxiety [37].However, Our epigenetic study of 5-HTTLPR did not consider its genotypes, which may make it hard to determine the real consistency of data. Further investigation is needed to examine the underlying mechanisms.

Apart from the shortcomings mentioned above, there were also some other limitations in our study. Firstly, we did not correct for multiple testing due to the exploratory nature, thus increasing the likelihood of receiving a significant result. Besides, we have to admit that the weight of HCs was normal and the effects of malnutrition on SLC6A4 methylation should be discussed. On the other hand, we did not consider other $\mathrm{CpG}$ units in the region that may be related to AN [30] and the environmental factors that may affect SLC6A4 methylation levels to a large extent [38]. Moreover, only a part of patients finished the follow-up treatment, which may account for the negative associations between 5-HTTLPR methylation and therapeutic responses, or the follow-up time was not enough due to the complexity of AN. Additionally, we did not detect the mRNA and 5-HT concentration that may be involved in the effect of DNA methylation mechanism. Recently, we have already recruited HCs with low weight, some limitations will be improved in our subsequent research.

\section{Conclusions}

Our study detected the methylation level of seven $\mathrm{CpG}$ units in 5-HTTLPR gene in patients with AN and found significant associations between them for the first time, supporting 5-HTTLPR methylation may be as a biomarker for AN but not a biomarker to distinguish the two subtypes of AN and predict the therapeutic responses. The discovery provides direction for the epigenetic mechanisms of AN and may offer a new perspective for targeted therapy of AN.

\section{Abbreviations}

AN: Anorexia Nervosa; ED: Eating Disorder; HC: healthy controls; AN-R: restricting type; AN-BP: binge/purging type; SLC6A4: serotonin transporter gene; 5-HTT: serotonin transporter; 5-HTTLPR: Serotonin Transporter Linked-Polymorphic Region; $n$ : Number of samples; Z: Z-Value; t: $t$ test; P: P-Value; ANOVA: one-way analysis of variance; $r$ : correlation coefficient; EAT-26: Eating Attitudes Test-26; EDE-Q 
6.0: Questionnaire Version of the Eating Disorders Examination; HAMD: Hamilton Depression Rating Scale; HAMA Hamilton Anxiety Scale; YB-OCS: Yale Brown Obsessive Compulsive Scale.

\section{Declarations}

\section{Acknowledgements}

We would like to appreciate all the participants that contributed to the study.

\section{Authors' contributions}

Q.-Q. He and Q. Kang performed the data analyses and wrote this article. S.-Y. Yu, S.-F Peng and H. Chen recruited the participants and revised this article. Z.-G Lin was responsible for laboratory testing. J. Chen and Z.-P Xiao designed this study. All authors read and approved the final manuscript.

\section{Funding}

This study was supported by the National Natural Science Foundation Program (81771461); Shanghai Municipal Health Commission (2019ZB0201); Shanghai clinical research center for mental health (SCRC$\mathrm{MH})$; Shanghai Clinical Medical Research Center for Psychiatric and Psychological Disorders (19MC1911100).

\section{Availability of data and materials}

The data and materials supporting the findings of this study are available from the corresponding author upon reasonable request.

\section{Ethics approval and consent to participate}

All procedures performed in studies involving human participants were in accordance with the ethical standards of the Ethics Committee of the Shanghai Mental Health Center (2020-32) and with the 1964 Helsinki declaration and its later amendments or comparable ethical standards. All participants signed the informed consent to the study.

\section{Consent for publication}

This article is original and has never been submitted or published elsewhere. All the authors have checked this final manuscript and approved to submit it.

\section{Competing interests}

The authors declare no conflict of interest.

\section{References}


1.Moore CA,Bokor BR.Anorexia Nervosa.Treasure Island (FL).2021.

2.Balasundaram P,Santhanam P.Eating Disorders.Treasure Island (FL).2021.

3.Yao S, Zhang R, Thornton LM, Peat CM, Qi B, Du S, et al. Screen-detected disordered eating and related traits in a large population sample of females in mainland China: China Health and Nutrition Survey. Int $J$ Eat Disord. 2021;54(1):24-35. https://doi.org/10.1002/eat.23409

4.Rozenblat V, Ong D, Fuller-Tyszkiewicz M, Akkermann K, Collier D, Engels R, et al. A systematic review and secondary data analysis of the interactions between the serotonin transporter 5-HTTLPR polymorphism and environmental and psychological factors in eating disorders. J Psychiatr Res. 2017;84:62-72. https://doi.org/10.1016/j.jpsychires.2016.09.023

5.Allis CD,Jenuwein T. The molecular hallmarks of epigenetic control. Nat Rev Genet. 2016;17(8):487-500. https://doi.org/10.1038/nrg.2016.59

6.Meaney MJ. Epigenetics and the biological definition of gene x environment interactions. Child Dev. 2010;81(1):41-79. https://doi.org/10.1111/j.1467-8624.2009.01381.x

7.Riva G. Neurobiology of Anorexia Nervosa: Serotonin Dysfunctions Link Self-Starvation with Body Image Disturbances through an Impaired Body Memory. Front Hum Neurosci. 2016;10:600. https://doi.org/10.3389/fnhum.2016.00600

8.Baker JH, Schaumberg K,Munn-Chernoff MA. Genetics of Anorexia Nervosa. Curr Psychiatry Rep. 2017;19(11):84. https://doi.org/10.1007/s11920-017-0842-2

9.Gelernter J, Pakstis AJ,Kidd KK. Linkage mapping of serotonin transporter protein gene SLC6A4 on chromosome 17. Hum Genet. 1995;95(6):677-80. https://doi.org/10.1007/bf00209486

10.Sundaramurthy D, Pieri LF, Gape H, Markham AF,Campbell DA. Analysis of the serotonin transporter gene linked polymorphism (5-HTTLPR) in anorexia nervosa. Am J Med Genet. 2000;96(1):53-5.

11.Ehrlich S, Franke L, Scherag S, Burghardt R, Schott R, Schneider N, et al. The 5-HTTLPR polymorphism, platelet serotonin transporter activity and platelet serotonin content in underweight and weight-recovered females with anorexia nervosa. Eur Arch Psychiatry Clin Neurosci. 2010;260(6):483-90.

https://doi.org/10.1007/s00406-009-0092-3

12.Wyler SC, Lord CC, Lee S, Elmquist JK,Liu C. Serotonergic Control of Metabolic Homeostasis. Front Cell Neurosci. 2017;11:277. https://doi.org/10.3389/fncel.2017.00277

13.Silverstein-Metzler MG, Shively CA, Clarkson TB, Appt SE, Carr JJ, Kritchevsky SB, et al. Sertraline inhibits increases in body fat and carbohydrate dysregulation in adult female cynomolgus monkeys. Psychoneuroendocrinology. 2016;68:29-38. https://doi.org/10.1016/j.psyneuen.2016.02.012 
14.Zhang K, Qu S, Chang S, Li G, Cao C, Fang K, et al. An overview of posttraumatic stress disorder genetic studies by analyzing and integrating genetic data into genetic database PTSDgene. Neurosci Biobehav Rev. 2017;83:647-656. https://doi.org/10.1016/j.neubiorev.2017.08.021

15.Caspi A, Sugden K, Moffitt TE, Taylor A, Craig IW, Harrington H, et al. Influence of life stress on depression: moderation by a polymorphism in the 5-HTT gene. Science. 2003;301(5631):386-9. https://doi.org/10.1126/science.1083968

16.Jang YJ, Lim SW, Moon YK, Kim SY, Lee H, Kim S, et al. 5-HTTLPR-rs25531 and Antidepressant Treatment Outcomes in Korean Patients with Major Depression. Pharmacopsychiatry. 2021,https://doi.org/10.1055/a-1478-4574

17.Tanahashi S, Tanii H, Konishi Y, Otowa T, Sasaki T, Tochigi M, et al. Association of Serotonin Transporter Gene (5-HTTLPR/rs25531) Polymorphism with Comorbidities of Panic Disorder. Neuropsychobiology. 2021;80(4):333-341. https://doi.org/10.1159/000512699

18.Li G, Wang L, Cao C, Fang R, Hall BJ, Elhai JD, et al. Post-traumatic stress symptoms of children and adolescents exposed to the 2008 Wenchuan Earthquake: A longitudinal study of 5-HTTLPR genotype main effects and gene-environment interactions. Int J Psychol. 2021;56(1):22-29. https://doi.org/10.1002/ijop.12614

19.Steiger H. Eating disorders and the serotonin connection: state, trait and developmental effects. J Psychiatry Neurosci. 2004;29(1):20-9.

20.Lee S, Kwok K, Liau C,Leung T. Screening Chinese patients with eating disorders using the Eating Attitudes Test in Hong Kong. Int J Eat Disord. 2002;32(1):91-7. https://doi.org/10.1002/eat.10064

21.Kang Q, Chan RCK, Li X, Arcelus J, Yue L, Huang J, et al. Psychometric Properties of the Chinese Version of the Eating Attitudes Test in Young Female Patients with Eating Disorders in Mainland China. Eur Eat Disord Rev. 2017;25(6):613-617. https://doi.org/10.1002/erv.2560

22.Chan CW,Leung SF. Validation of the Eating Disorder Examination Questionnaire: an online version. J Hum Nutr Diet. 2015;28(6):659-65. https://doi.org/10.1111/jhn.12275

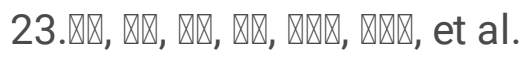

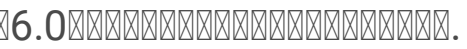
2017;31(05):350-355.

24.Nikolova YS, Koenen KC, Galea S, Wang CM, Seney ML, Sibille E, et al. Beyond genotype: serotonin transporter epigenetic modification predicts human brain function. Nat Neurosci. 2014;17(9):1153-5. https://doi.org/10.1038/nn.3778

25.Chiarella J, Schumann L, Pomares FB, Frodl T, Tozzi L, Nemoda Z, et al. DNA methylation differences in stress-related genes, functional connectivity and gray matter volume in depressed and healthy adolescents. J Affect Disord. 2020;271:160-168. https://doi.org/10.1016/j.jad.2020.03.062 
26.Lam D, Ancelin M-L, Ritchie K, Freak-Poli R, Saffery R,Ryan J. Genotype-dependent associations between serotonin transporter gene (SLC6A4) DNA methylation and late-life depression. BMC Psychiatry. 2018;18(1)https://doi.org/10.1186/s12888-018-1850-4

27.Sales AJ, Guimaraes FS,Joca SRL. DNA methylation in stress and depression: from biomarker to therapeutics. Acta Neuropsychiatr. 2021;33(5):217-241. https://doi.org/10.1017/neu.2021.18

28.Schiele MA, Zwanzger P, Schwarte K, Arolt V, Baune BT,Domschke K. Serotonin Transporter Gene Promoter Hypomethylation as a Predictor of Antidepressant Treatment Response in Major Depression: A Replication Study. Int J Neuropsychopharmacol. 2021;24(3):191-199.

https://doi.org/10.1093/ijnp/pyaa081

29.Pjetri E, Dempster E, Collier DA, Treasure J, Kas MJ, Mill J, et al. Quantitative promoter DNA methylation analysis of four candidate genes in anorexia nervosa: a pilot study. J Psychiatr Res. 2013;47(2):280-2. https://doi.org/10.1016/j.jpsychires.2012.10.007

30.Boehm I, Walton E, Alexander N, Batury V-L, Seidel M, Geisler D, et al. Peripheral serotonin transporter DNA methylation is linked to increased salience network connectivity in females with anorexia nervosa. Journal of Psychiatry and Neuroscience. 2020;45(3):206-213. https://doi.org/10.1503/jpn.190016

31.Eddy KT, Keel PK, Dorer DJ, Delinsky SS, Franko DL,Herzog DB. Longitudinal comparison of anorexia nervosa subtypes. Int J Eat Disord. 2002;31(2):191-201. https://doi.org/10.1002/eat.10016

32.Castellini G, Ricca V, Lelli L, Bagnoli S, Lucenteforte E, Faravelli C, et al. Association between serotonin transporter gene polymorphism and eating disorders outcome: A 6-year follow-up study. American Journal of Medical Genetics Part B: Neuropsychiatric Genetics. 2012;159B(5):491-500. https://doi.org/10.1002/ajmg.b.32052

33.Webb LM, Phillips KE, Ho MC, Veldic M,Blacker CJ. The Relationship between DNA Methylation and Antidepressant Medications: A Systematic Review. Int J Mol Sci.

2020;21(3)https://doi.org/10.3390/ijms21030826

34.Iga J, Watanabe SY, Numata S, Umehara H, Nishi A, Kinoshita M, et al. Association study of polymorphism in the serotonin transporter gene promoter, methylation profiles, and expression in patients with major depressive disorder. Hum Psychopharmacol. 2016;31(3):193-9.

https://doi.org/10.1002/hup.2527

35.Okada S, Morinobu S, Fuchikami M, Segawa M, Yokomaku K, Kataoka T, et al. The potential of SLC6A4 gene methylation analysis for the diagnosis and treatment of major depression. J Psychiatr Res. 2014;53:47-53. https://doi.org/10.1016/j.jpsychires.2014.02.002

36.Gaunt TR, Shihab HA, Hemani G, Min JL, Woodward G, Lyttleton O, et al. Systematic identification of genetic influences on methylation across the human life course. Genome Biol. 2016;17:61. 
37.Schneider I, Kugel H, Redlich R, Grotegerd D, Burger C, Burkner PC, et al. Association of Serotonin Transporter Gene AluJb Methylation with Major Depression, Amygdala Responsiveness, 5HTTLPR/rs25531 Polymorphism, and Stress. Neuropsychopharmacology. 2018;43(6):1308-1316. https://doi.org/10.1038/npp.2017.273

38.Gottschalk MG, Domschke K,Schiele MA. Epigenetics Underlying Susceptibility and Resilience Relating to Daily Life Stress, Work Stress, and Socioeconomic Status. Front Psychiatry. 2020;11:163. https://doi.org/10.3389/fpsyt.2020.00163

\section{Tables}

Table 1 Demographic and clinical characteristics between AN and HC group

\begin{tabular}{|lllll|}
\hline & AN $(\mathrm{n}=91)$ & $\mathrm{NC}(\mathrm{n}=87)$ & $t$ & $p$ \\
\hline Age (years) & $18.96 \pm 4.14$ & $19.95 \pm 3.74$ & -1.850 & 0.068 \\
\hline Age of onset (years) & $16.5 \pm 2.64$ & $/$ & $/$ & $/$ \\
\hline Diease course (months) & $15(132,4)$ & $/$ & $/$ & $/$ \\
\hline Education level (years) & $12.18 \pm 2.94$ & $12.99 \pm 2.67$ & -1.876 & 0.062 \\
\hline Current BMI (kg/m $\left.{ }^{2}\right)$ & $15.22 \pm 2.08$ & $20.02 \pm 3.20$ & -13.13 & 0.000 \\
\hline maximum body weight $(\mathrm{kg})$ & $55.23 \pm 7.94$ & $55.50 \pm 8.74$ & -0.228 & 0.820 \\
\hline Maximum BMI (kg/m $\left.{ }^{2}\right)$ & $21.06 \pm 3.12$ & $20.76 \pm 3.49$ & 0.628 & 0.531 \\
\hline Ideal weight $(\mathrm{kg})$ & $44.79 \pm 4.15$ & $49.96 \pm 4.89$ & -8.077 & $\mathbf{0 . 0 0 0}$ \\
\hline Ideal BMI (kg/m $\left.{ }^{2}\right)$ & $17.06 \pm 1.30$ & $18.65 \pm 2.31$ & -5.979 & 0.000 \\
\hline HAMD total scale & $14.32 \pm 6.74$ & $/$ & & \\
\hline HAMA total scale & $11.25 \pm 7.69$ & $/$ & $/$ & $/$ \\
\hline YB-OCS total scale & $17.51 \pm 10.99$ & $/$ & $/$ & $/$ \\
\hline EAT-26 total scale & $25.06 \pm 17.30$ & $3.97 \pm 4.01$ & 12.332 & $\mathbf{0 . 0 0 0}$ \\
\hline EDE-Q6.0 total scale & $2.33 \pm 1.47$ & $0.74 \pm 0.74$ & 9.984 & $\mathbf{0 . 0 0 0}$ \\
\hline
\end{tabular}

Note $\mathbb{1}^{1}$ The disease course was not normally distributed, and the median (maximum and minimum) was used for statistical description. 
$P$. t-test

Table 2 correlation analysis of 5-HTTLPR methylation level of and clinical symptoms in AN subtypes

\begin{tabular}{|lllllll|}
\hline CpG units & \multicolumn{2}{l}{ AN-R(n=44) } & \multicolumn{3}{l|}{ AN-BP( $\mathrm{n}=47)$} \\
& BMI & EAT-26 & EDE-Q6.0 & BMI & EAT-26 & EDE-Q6.0 \\
& & total score & $\begin{array}{l}\text { total score } \\
\text { total score }\end{array}$ & & total score \\
\hline 1 & 0.139 & 0.044 & 0.031 & $0.350^{*}$ & -0.121 & 0.031 \\
\hline 11 & 0.139 & 0.054 & 0.237 & 0.073 & 0.064 & 0.138 \\
\hline 24.25 & 0.065 & $-0.325^{*}$ & $-0.338^{*}$ & 0.179 & 0.113 & 0.223 \\
\hline CPG_mean & -0.027 & -0.282 & -0.285 & 0.213 & 0.043 & 0.117 \\
\hline
\end{tabular}

Note $邓$ r: correlation coefficient; $* P<0.05, * \star P<0.01$

Table 3 The change of 5-HTTLPR methylation level after treatment in AN patients

\begin{tabular}{|lllll|}
\hline CpG units & Basement & Twelfth week & $Z^{1}$ & $p$ \\
\hline 1 & $9.21 \pm 3.22$ & $9.42 \pm 5.11$ & -0.130 & 0.898 \\
\hline 11 & $5.79 \pm 2.39$ & $4.63 \pm 2.09$ & 1.615 & 0.106 \\
\hline 24.25 & $1.84 \pm 1.57$ & $2.26 \pm 2.82$ & 0.176 & 0.860 \\
\hline 26.27 .28 & $5.74 \pm 2.28$ & $5.42 \pm 1.61$ & 0.516 & 0.612 \\
\hline 31.32 & $7.11 \pm 2.51$ & $6.47 \pm 3.91$ & 0.574 & 0.573 \\
\hline 33.34 .35 .36 & $4.84 \pm 1.46$ & $4.74 \pm 2.42$ & 0.166 & 0.870 \\
\hline CpG_mean & $5.75 \pm 1.01$ & $5.49 \pm 1.53$ & 0.860 & 0.420 \\
\hline
\end{tabular}

Note $]^{1}$ Repeated measurement ANOVA was conducted, and the data did not meet the spherical symmetry. Using the corrected results of Huynh-Feldt as statistical test.

\section{Figures}




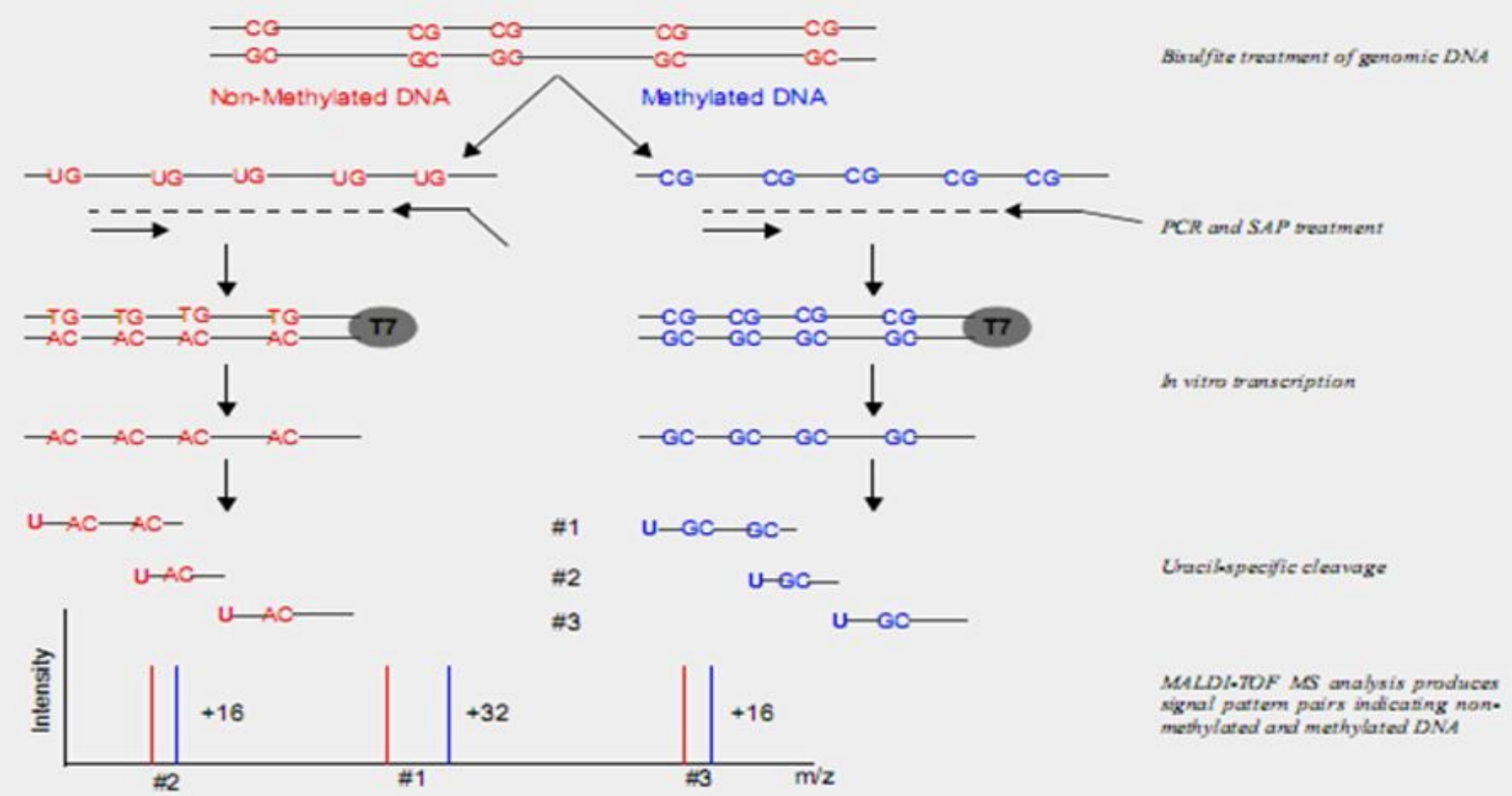

Figure 1: Overview of EpiTYPERTM assay

Figure 1

The specific principle of 5-HTTLPR DNA methylation conducted by the EpiTYPER software.

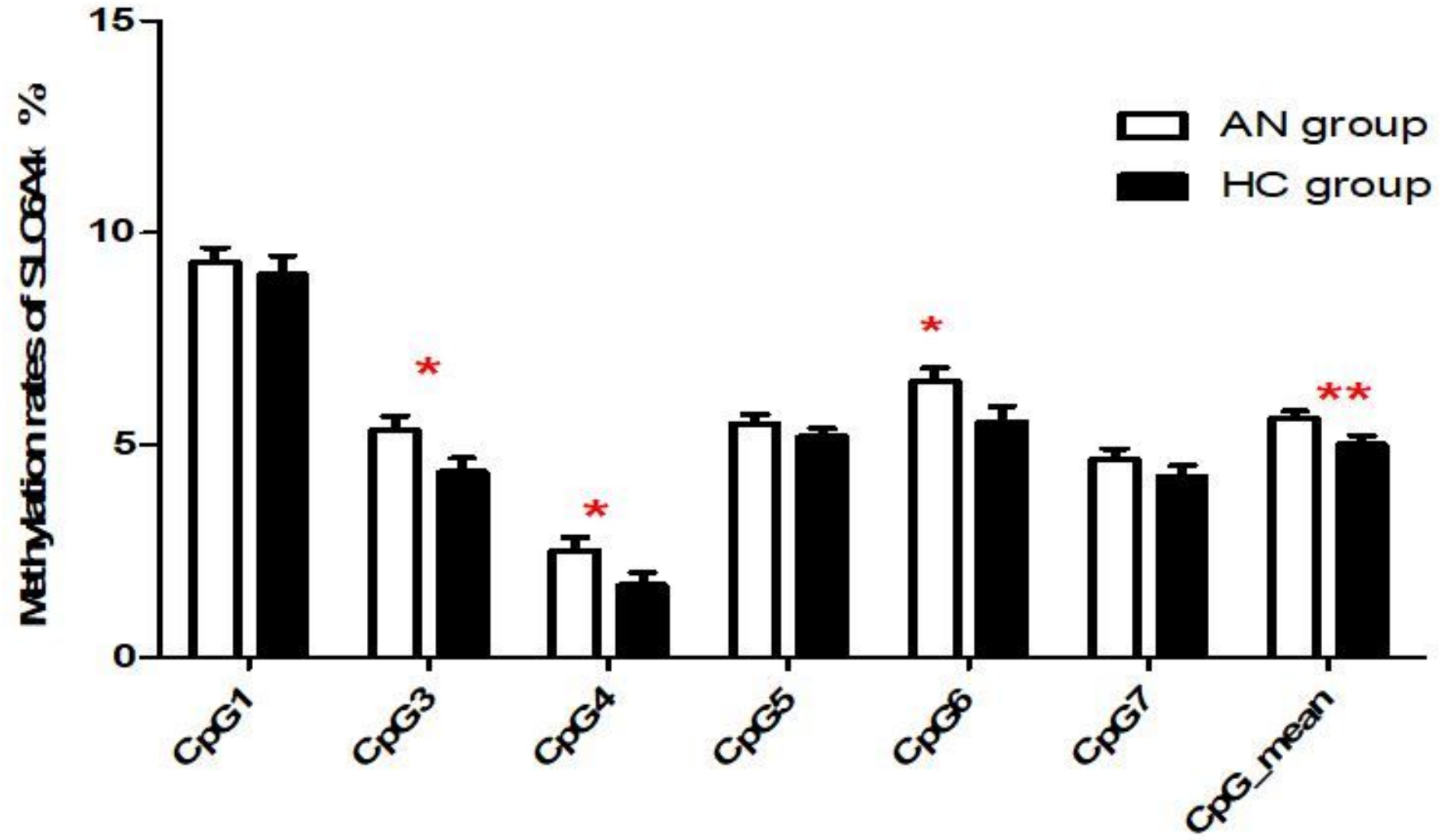


Figure 2

Comparison of 5-HTTLPR methylation rates at each $\mathrm{CpG}$ site between AN and healthy controls Noteđ* $P<0.05, * \star P<0.01$

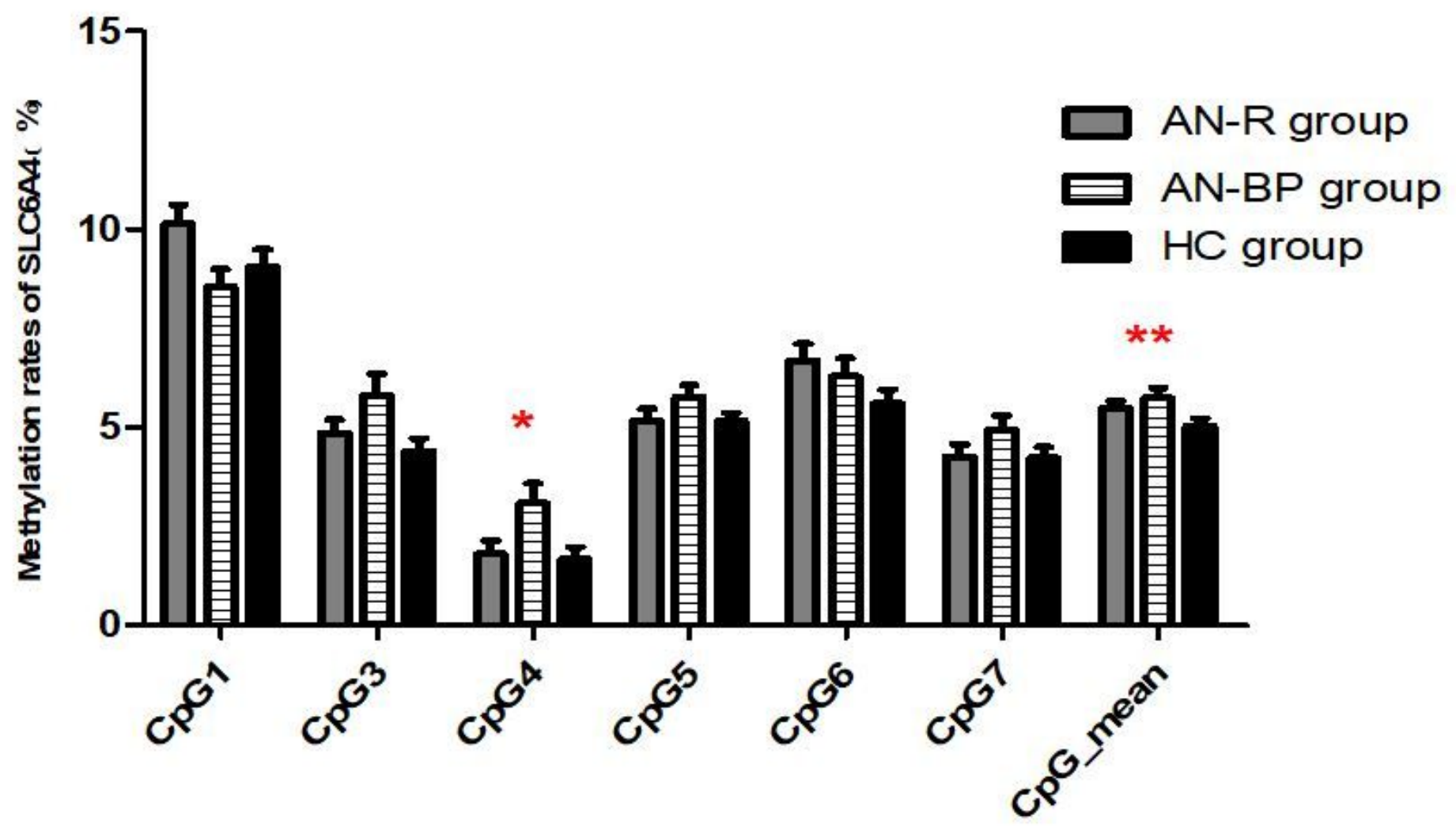

Figure 3

Comparison of 5-HTTLPR methylation rates at each CpG site among AN-R group, AN-BP group and healthy controls

Note $\rrbracket^{\star} P<0.05, * \star P<0.01$ 